\title{
The recent results from the annual modulation analysis of the XMASS-I dark matter data
}

\author{
Byeongsu Yang ${ }^{* 1,2}$ for XMASS collaboration \\ ${ }^{1}$ Kamioka Observatory, Institute for Cosmic Ray Research, the University of Tokyo \\ Higashi-Mozumi, Kamioka, Hida, Gifu, 506-1205, Japan \\ ${ }^{2}$ Kavli Institute for the Phsics and Mathematics of the Universe(WPI), the University of Tokyo \\ Kashiwa, CHiba, 277-8582, Japan \\ E-mail: byang@suketto.icrr.u-tokyo.ac.jp
}

\begin{abstract}
XMASS-I is dedicated to a direct dark matter search using nearly 1 tons of liquid xenon as target material. One of the signatures of dark matter in the direct detection experiments is the annual modulation of the event rate due to the relative motion of the Earth around the Sun. We have continuously collected data with a low trigger threshold of 0.6 keVee for more than 3 years since November 2013. In this talk, the recent results from the annual modulation analysis using full-volume of the XMASS-I detector will be shown.
\end{abstract}

35th International Cosmic Ray Conference

10-20 July, 2017

Bexco, Busan, Korea

${ }^{*}$ Speaker. 


\section{Introduction}

XMASS experiment is a multi purpose experiment with large volume of liquid Xenon. The XMASS collaboration is consists of $\sim 40$ physicists from 10 institutes of Japan and Korea (Japan 8, Korea 2), and and purposes to detect dark matter directly, to observe low energy solar neutrino such as $\mathrm{pp} /{ }^{7} \mathrm{Be}$ and to search for neutrino-less double beta decay[1].

XMASS-I is dedicated to a direct dark matter search. Its detector is located at the Kamioka Observatory (overburden 2700 m.w.e) in Japan. The detailed design and performance are described in [2]. The detector is immersed in a water tank, $10 \mathrm{~m}$ in diameter and $10.5 \mathrm{~m}$ in height, which is equipped with 72 Hamamatsu H3600 photomultiplier tubes (PMTs), and acts as an outer detector for active muon veto and a passive radiation shield against neutrons and gamma rays from the surrounding rock. 642 high quantum efficiency (28-40\% at $175 \mathrm{~nm})$ Hamamatsu R10789 PMTs are mounted in the liquid xenon detector, an approximate sphere with an average radius of $40 \mathrm{~cm}$. It have high light yield $(14.7 \mathrm{pe} / \mathrm{keV})$ and low threshold $(0.6 \mathrm{keVee})$ and sensitive to $e / \gamma$-ray events as well as nuclear recoil. In December 2010, XMASS-I started data taking, and we refurbished the detector from August 2012 to October 2013, and data taking was resumed, in November 2013. Now, the 4th year continuity operation is ongoing.

The well-known phenomenon of dark matter signal is the annual modulation of event rate due to relative motion of the Earth around the Sun. It would be a strong signature of dark matter. The most significant result is that of the DAMA/LIBRA experiment at the Gran Sasso National Laboratory in Italy which indicated an annual modulation signature [3]. The DAMA/LIBRA experiment reported an observation of event rate annual modulation with a $9 \sigma$ significance in 1.33 ton-year of data taken over 14 annual cycles with 100 to $250 \mathrm{~kg}$ of NaI(Tl) detectors. Using $\sim 1.3$ years data, we published our results on direct dark matter search by annual modulation[4]. Recently, we analyzed new $\sim 1.3$ years data and the result will be shown.

\section{Data Analysis}

In the previous paper[4], we analyzed the data taken between November 2013 and March 2015. In this analysis we added the data set taken between April 2015 and July 2016. Hereafter, let the former period Run1 and the later period Run2. Like the previous paper, we selected periods with stable temperature (172.6-173.0 K) and pressure of Xe (0.162 - $0.164 \mathrm{MPa}$ absolute). For only Run2, we kept more stable temperature (172.6-172.9 K) and pressure of Xe (0.162 - 0.163 $\mathrm{MPa}$ absolute). After removing periods of operation with excessive PMT noise or data acquisition problems, the total live time and exposure became 800.0 and 1.82 ton year. The live time of Run 1 and Run2 are 387.8 and 412.2 days, respectively. By the way, we recovered a part of bad runs of in Run1, and about 28.6 days data increased compared with the data set of the previous paper[4].

Event selection is almost same with the previous analysis. The difference is a selction to remove surface background[5]. In the former analysis, background events that occurred in front of PMT window were removed using the values of 'Max-photoelectron/Total-photoelectron' where Max-photoelectron and Total-photoelectron are the largest photoelectron counts in one PMT among all PMTs and the total number of photoelectrons in the event. In this analysis, to remove surface background that occurred near the detector wall as well as in front of PMT window, maxi- 

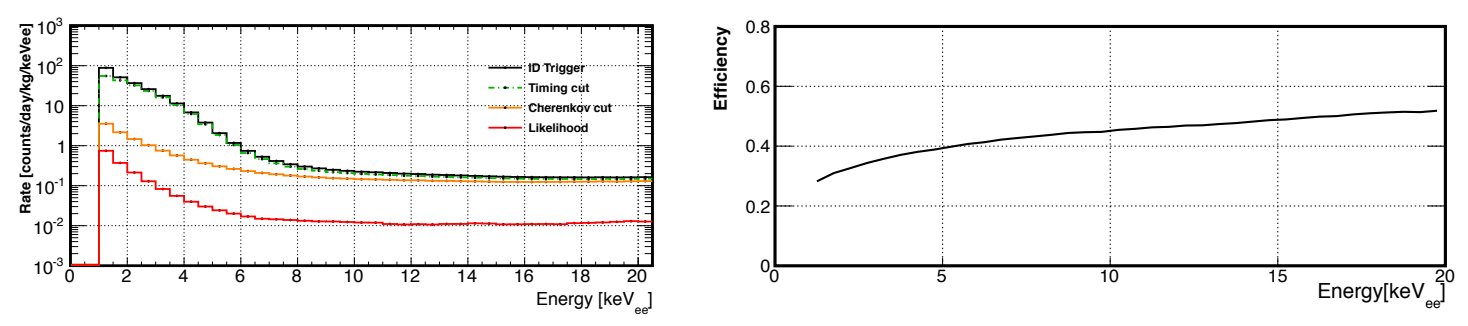

Figure 1: Energy spectrum after each event selection for total exposure (left). Total cut efficiency for uniformly distributed signal events after all cuts. (right).

mum likelihood method is introduced. The likelihood is the function of 'Max-photoelectron/Totalphotoelectron', sphericity and aplanarity. They are constructed based on PE hits pattern. The left and right figure of the Fig. 1 showed the energy spectrum after each event selection for total exposure and the total cut efficiency after all the selections, respectively. The efficiency was evaluated from electron Monte Carlo simulation with a flat energy spectrum uniformly distributed in the sensitive volume. The count rate for the data and the cut efficiency after all the selections are $\sim 0.75$ $(\sim 0.028)$ events $/ \mathrm{day} / \mathrm{kg} / \mathrm{keV}_{\text {ee }}$ and $\sim 30 \%(\sim 40 \%)$ at $1.0(5.0) \mathrm{keV}_{\text {ee. }}$. Compared with the previous analysis, $30 \%$ reduction of data with similar efficiency around $1.0 \mathrm{keV}_{\mathrm{ee}}$ are achieved thanks to the improved event selection.

The ${ }^{57} \mathrm{Co}$ calibration data have been taken at from $z=-40 \mathrm{~cm}$ to $+40 \mathrm{~cm}$ along the center vertical axis of the detector to track photoelectron yield and optical properties of the liquid xenon [2]. A difference of about $10 \%$ was observed as the position dependence for this photoelectron yield. The Fig. 2 showed the stability of photoelectron yield. The photoelectron yield during the Run 1 were spread with RMS of $2.4 \%$. It changed gradually from the beginning of the Run1, suddenly dropped at the power failure of August 2014 and rapidly changed at swap of cold heads in XMASS refrigerator from December 2014 to February 2015. According to Monte Carlo simulation, those

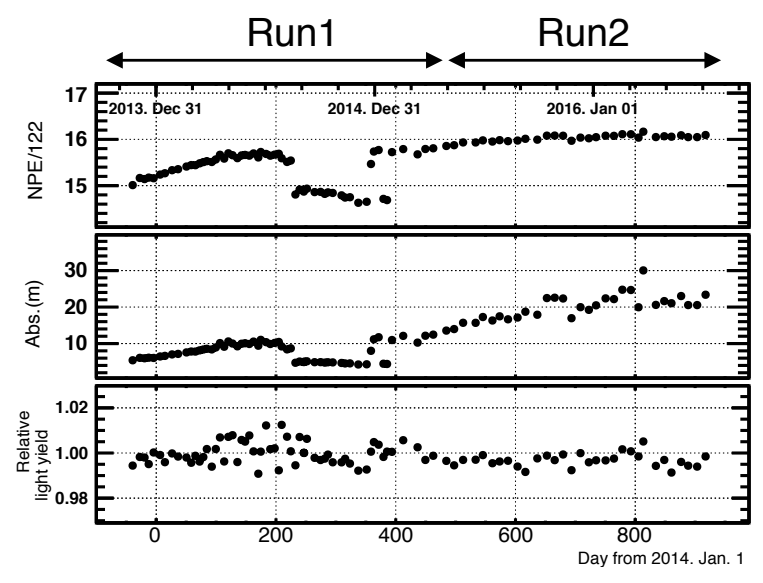

Figure 2: Light yield stability was monitored with a ${ }^{57} \mathrm{Co} 122 \mathrm{keV}$ gamma ray source.(top) The scintillation light absorption length in xenon (middle) and the relative intrinsic scintillation light yield $\left(R_{\text {yield }}\right)$ (bottom) was obtained by comparing calibration data with the Monte Carlo simulation by considering optical parameters such as absorption and scattering length. 

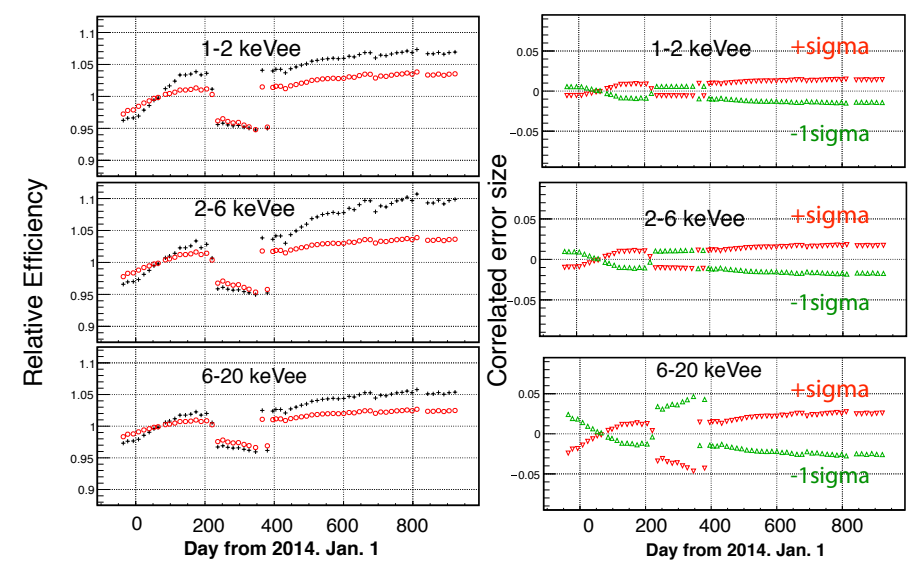

Figure 3: Relative efficiencies for both signal (cross) and background (circle) by normalizing the overall efficiency at an absorption length of $8 \mathrm{~m}$ for different energy ranges (left). Size of correlated uncertainties due to the position dependence or background model are also shown with $\pm 1 \sigma$ (right). Their changes are also normalized at same absorption length.

change can be explained change of the scintillation light absorption length in xenon. We thought that gaseous impurity caused the changes, and the gas circulation was started to remove the impurity in March 2015. Then its RMS of Run2 reduced by $0.5 \%$. The relative intrinsic light yield of the liquid xenon scintillator is extracted from the ${ }^{57} \mathrm{Co}$ calibration data and the Monte Carlo simulation [2]. From April 2014 to September 2014, they were spread about 2 times more, because gain calibration method of the the waveform digitizers (CAEN V1751) in the period was different from that in other periods. Its RMS over Run 1 and Run 2 are $\sim 0.6 \%$ and $\sim 0.3 \%$, respectively.

Systematic errors associated with the photoelectron yield changes during exposure were treated same way as described in [4]. As the time dependence of the photoelectron yield affects the efficiency of the cuts, we evaluate the absorption length dependence of the relative cut efficiencies through Monte Carlo simulation. If we normalize the overall efficiency at an absorption length of $8 \mathrm{~m}$, this efficiency changes from $-4 \%$ to $+8 \%$ over the relevant absorption range. The position dependence of the efficiency was taken into account as a correlated systematic error $(\sim \pm 2.5 \%)$. This is the dominant systematic uncertainty in the present analysis. The other systematic errors are same as previous analysis.[4] Compared with Run1, the systematic uncertainty of Run1 is smaller, because the time dependence of the photoelectron yield and gain instability of the waveform digitizers are much stable.

To retrieve the annual modulation amplitude from the data, the least squares method for the time-binned data was used. The data set was divided into 63 time-bins $\left(t_{\text {bins }}\right)$ with roughly 15 days of real time each. The data in each time-bin were then further divided into energy-bins $\left(E_{b i n s}\right)$ with a width of $0.5 \mathrm{keV}_{\text {ee. }}$ A 'pull term' method was used to fit all energy- and time-bins simultaneously to treat the correlated errors.

\section{Results and discussion}

We performed two analyses, one assuming WIMP interactions and the other independent of 
any specific dark matter model. Hereafter we call the former case the WIMP analysis and the latter a model independent analysis. In the case of the WIMP analysis, $\chi^{2}$ defined as:

$$
\chi^{2}=\sum_{i}^{E_{\text {bins }}} \sum_{j}^{t_{\text {bins }}}\left(\frac{\left(R_{i, j}^{\mathrm{data}}-R_{i, j}^{\mathrm{ex}}(\alpha, \beta)\right)^{2}}{\sigma(\mathrm{stat})_{i, j}^{2}+\sigma(\mathrm{sys})_{i, j}^{2}}\right)+\alpha^{2}+\sum^{N s y s} \beta_{i}^{2},
$$

where $R_{i, j}^{\text {data }}, R_{i, j}^{\text {ex }}, \sigma(\text { stat })_{\mathrm{i}, \mathrm{j}}$ and $\sigma(\mathrm{sys})_{\mathrm{i}, \mathrm{j}}$ are data, expected event rate, statistical and systematic error, respectively, of the $i$-th energy and $j$-th time bin. The time is denoted as the number of days from January 1, 2014. A penalty term $\alpha$ represents the size of the relative efficiency error and it is common for all fitted energy bins, therefore, the size of error changes simultaneously during fit procedure. $\alpha=1(-1)$ corresponds to $1 \sigma(-1 \sigma)$ correlated systematic error on the expected event rate in that energy bin as shown in Fig. 3. Other pull-terms $\beta_{i}$ are parameters for systematical uncertainty of expected WIMP signal simulation. Here, two systematical uncertainties, scintillation efficiency for nuclear recoil (Leff)[6] and the time constant of nuclear recoil are considered. The expected signals are simulated with those errors to estimate the impact to amplitude $A_{i}^{s}(\beta)$ and unmodulated component $C_{i}^{S}(\beta)$. Those errors are taken into account by the pull term $\beta_{i}$.

The expected modulation amplitudes become a function of the WIMP mass $A_{i}\left(m_{\chi}\right)$ as the WIMP mass $m_{\chi}$ determines the recoil energy spectrum. The expected rate in a bin then becomes:

$$
R_{i, j}^{\mathrm{ex}}=\int_{t_{j}-\frac{1}{2} \Delta t_{j}}^{t_{j}+\frac{1}{2} \Delta t_{j}}\left(\varepsilon_{i}^{b}(\alpha) \cdot\left(B_{i} t+C_{i}^{b}\right)+\sigma_{\chi n} \cdot \varepsilon_{i}^{s}(\alpha) \cdot\left(C_{i}^{S}(\beta)+A_{i}^{s}(\beta) \cos 2 \pi \frac{\left(t-t_{0}\right)}{T}\right)\right) d t
$$

where $\sigma_{\chi n}$ is the WIMP-nucleon cross section, $\varepsilon_{i}^{b}(\alpha)$ and $\varepsilon_{i}^{s}(\alpha)$ are the relative efficiency for background and signal, respectively. The background component was described by the linear function, $B_{i}$ for slope and $C_{i}^{b}$ constant for the background in $i$-th bin. $A_{i}^{s}(\beta)$ represents an amplitude and $C_{i}^{s}(\beta)$ for unmodulated component for signal in $i$-th bin. To obtain the WIMP-nucleon cross section the data was fitted in the energy range of $1.0-20 \mathrm{keV}_{\mathrm{ee}}$. We assume a standard spherical isothermal galactic halo model with the most probable speed of $v_{0}=220 \mathrm{~km} / \mathrm{s}$, the Earth's velocity relative to the dark matter distribution of $v_{E}=232+15 \sin 2 \pi\left(t-t_{0}\right) / T \mathrm{~km} / \mathrm{s}$, and a galactic escape

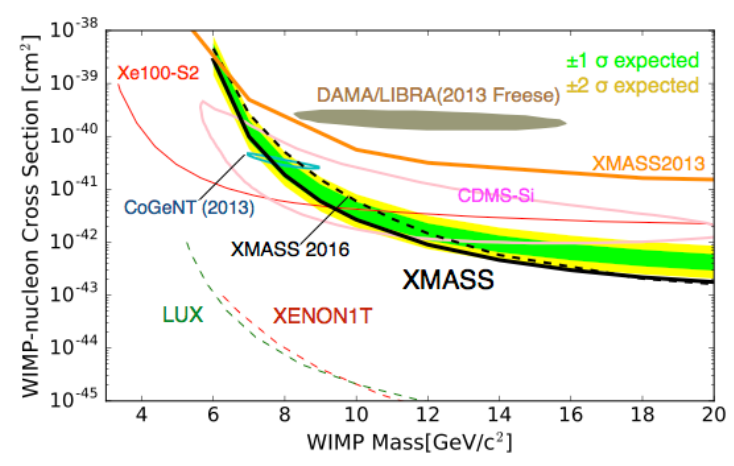

Figure 4: Limits on the spin-independent elastic WIMP-nucleon cross section as a function of WIMP mass. The solid line shows the XMASS $90 \%$ C.L. exclusion from the annual modulation analysis. The $\pm 1 \sigma$ and $\pm 2 \sigma$ bands represent the expected $90 \%$ exclusion distributions. Limits as well as allowed regions from other searches based on counting method are also shown $[9,10,11,13,14,15]$. 


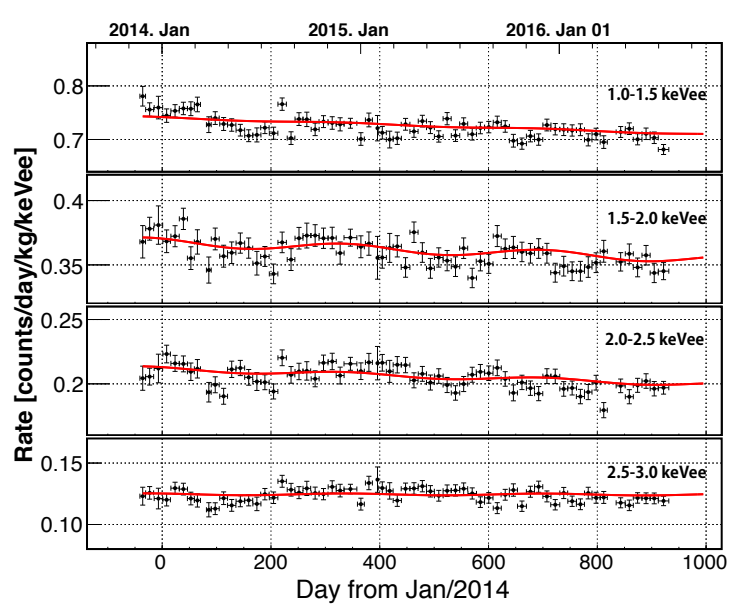

Figure 5: Observed count rate as a function of time in the $1.0-3.0 \mathrm{keV}_{\mathrm{ee}}$ energy range. The black error bars show the statistical uncertainty of the count rate. The solid curves represent the best fit result for model independent analysis before total efficiency correction.

velocity of $v_{\text {esc }}=544 \mathrm{~km} / \mathrm{s}$ [7], a local dark matter density of $0.3 \mathrm{GeV} / \mathrm{cm}^{3}$, following [8]. In the analysis, the signal efficiencies for each WIMP mass are estimated from Monte Carlo simulation of uniformly distributed nuclear recoil events in the liquid xenon volume.

As we found no significant signal, the $90 \%$ C.L. upper limit on the WIMP-nucleon cross section is shown in Fig. 4. The exclusion upper limit of $1.9 \times 10^{-41} \mathrm{~cm}^{2}$ at $8 \mathrm{GeV} / \mathrm{c}^{2}$ was obtained. To evaluate the sensitivity of WIMP-nucleon cross section, we carried out a statistical test by applying the same analysis to 10,000 dummy samples with the same statistical and systematic errors as data but without modulation by the same procedure of [4]. At first, the time-averaged energy spectrum was obtained from the observed data. Then, we performed a toy Monte Carlo simulation to simulate time variation of event rate of background at each energy bin assuming the same live time as data and including systematic uncertainties. The $\pm 1 \sigma$ and $\pm 2 \sigma$ bands in Fig. 4 outline the expected $90 \%$ C.L. upper limit band for the no-modulation hypothesis using the dummy samples. The result excludes the $3 \sigma$ DAMA/LIBRA allowed region as interpreted in [9].

For the model independent analysis, the expected event rate was estimated as:

$$
\begin{gathered}
\chi^{2}=\sum_{i}^{E_{\text {bins }} t_{\text {bins }}} \sum_{j}^{\left(R_{i, j}^{\mathrm{data}}-R_{i, j}^{\mathrm{ex}}\right)^{2}}\left(\frac{\mathrm{stat}_{i, j}^{2}+\sigma(\mathrm{sys})_{i, j}^{2}}{\sigma(\mathrm{a})}+\alpha^{2},\right. \\
R_{i, j}^{\mathrm{ex}}=\int_{t_{j}-\frac{1}{2} \Delta t_{j}}^{t_{j}+\frac{1}{2} \Delta t_{j}}\left(\varepsilon_{i}^{s} a_{i} \cos 2 \pi \frac{\left(t-t_{0}\right)}{T}+\varepsilon_{i}^{b}(\alpha)\left(B_{i} t+C_{i}^{b}\right)\right) d t,
\end{gathered}
$$

where the free parameters $C_{i}$ and $A_{i}$ were the unmodulated event rate and the modulation amplitude, respectively. $t_{0}$ and $T$ were the phase and period of the modulation, and $t_{j}$ and $\Delta t_{j}$ was the timebin's center and width, respectively. In the fitting procedure, the $1.0-20 \mathrm{keV}_{\text {ee }}$ energy range was used and the modulation period $T$ was fixed to one year and the phase $t_{0}$ to 152.5 days $(\sim 2 \mathrm{nd}$ of June) when the Earth's velocity relative to the dark matter distribution is expected to be maximal.

Fig. 6 shows the best fit amplitudes as a function of energy for 'pull term' after correcting the efficiency(Fig. 1 (right)). As the previous paper[4], we find a slight negative amplitude below 


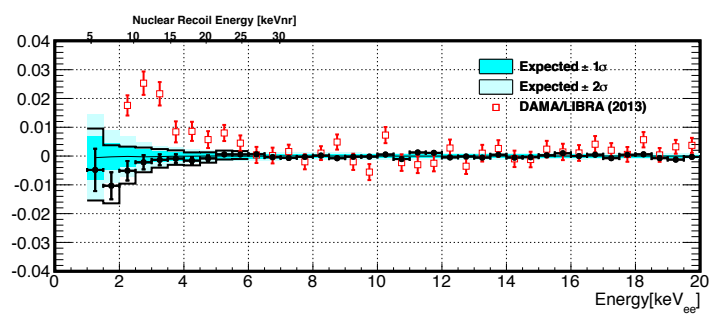

Figure 6: Modulation amplitude as a function of energy for the model independent analyses using the "pull term' method (solid circle). Solid lines represent $90 \%$ positive (negative) upper limits on the amplitude. The $\pm 1 \sigma$ and $\pm 2 \sigma$ bands represent the expected amplitude region (see detail in the text). DAMA/LIBRA result (square) is also shown [3].

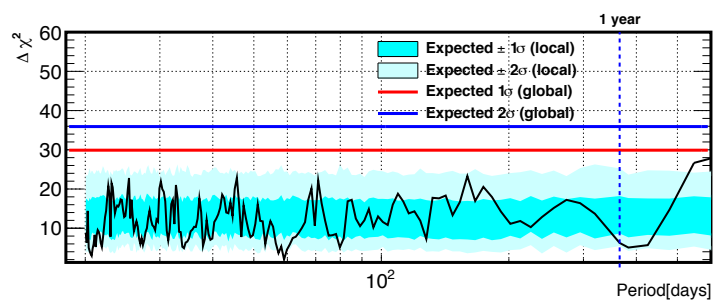

Figure 7: The power spectrum between 20 and 600 days frequency for $1-6 \mathrm{keV}_{\mathrm{ee}}$ energy range together with significance level by the test statistics with dummy samples.

$3 \mathrm{keV}_{\text {ee }}$. The $\pm 1 \sigma$ and $\pm 2 \sigma$ bands in Fig. 6 represent expected amplitude coverage derived from same dummy sample above. This test gave a $p$-value of $0.11(1.6 \sigma)$. The significance for modulation hypothesis is smaller than the previous analysis[4] (p-val of 0.014 for 'pull-term' method) after adding more than one year cycle of data. To be able to test any model of dark matter, we evaluated the constraints on the positive and negative amplitude separately in Fig. 6. The upper limits on the amplitudes in each energy bin were calculated by considering only regions of positive or negative amplitude. They were calculated by integrating Gaussian distributions based on the mean and sigma of data $(=G(a))$ from zero. The positive or negative upper limits are satisfied with 0.9 for $\int_{0}^{a_{u p}} G(a) d a / \int_{0}^{\infty} G(a) d a$ or $\int_{a_{u p}}^{0} G(a) d a / \int_{-\infty}^{0} G(a) d a$, where $a$ and $a_{u p}$ are the amplitude and its $90 \%$ C.L. upper limit, respectively. The 'pull term' method obtained positive (negative) upper limit of $0.96(-1.5) \times 10^{-2}$ events/day $/ \mathrm{kg} / \mathrm{keV}_{\mathrm{ee}}$ between 1.0 and $1.5 \mathrm{keV}_{\mathrm{ee}}$ and the limits become stricter at higher energy. The energy resolution $(\sigma / \mathrm{E})$ at $1.0(5.0) \mathrm{keV}_{\text {ee }}$ is estimated to be $36 \%$ (19\%) comparing gamma ray calibrations and its Monte Carlo simulation. As a guideline, we make direct comparisons with other experiments not by considering a specific dark matter model but amplitude count rate. The modulation amplitude of $\sim 2 \times 10^{-2}$ events $/ \mathrm{day} / \mathrm{kg} / \mathrm{keV}$ ee between 2.0 and $3.5 \mathrm{keV}_{\mathrm{ee}}$ was obtained by DAMA/LIBRA [3] and XENON100 reported $1.67 \pm 0.73 \times 10^{-3}$ events/day $/ \mathrm{kg} / \mathrm{keV}_{\text {ee }}\left(2.0-5.8 \mathrm{keV}_{\mathrm{ee}}\right)$ [16]. XMASS obtained $90 \%$ C.L positive upper limits of $(1.3-3.2) \times 10^{-3}$ events $/ \mathrm{day} / \mathrm{kg} / \mathrm{keV}_{\text {ee }}$ in same energy region and gives the more stringent constraint. This fact is important when we test the dark matter model.

To find any period in the data, the power spectrum analysis was also performed with treating the phase $t_{0}$ free parameter in the energy range between $1-6 \mathrm{keV}_{\text {ee }}$ For each period, $\Delta \chi^{2}$ between null and periodic hypotheses was calculated for both data and the dummy samples for local sig- 
nificance $\pm 1 \sigma$ and $\pm 2 \sigma$. The global significance was evaluated by the maximum $\Delta \chi^{2}$ in the calculated range in each dummy sample to avoid 'look somewhere else' effect. No significant period was found between 20 and 600 days.

\section{Conclusions}

XMASS with its large exposure and high photoelectron yield (low energy threshold) conducted an annual modulation search. For the WIMP analysis, the exclusion upper limit of $1.9 \times 10^{-41} \mathrm{~cm}^{2}$ at $8 \mathrm{GeV} / \mathrm{c}^{2}$ was obtained and the result excludes the DAMA/LIBRA allowed region for WIMP masses higher than that. In the case of the model independent case, the analysis was carried out from the energy threshold of $1.0 \mathrm{keV}_{\text {ee }}$ which is lower than DAMA/LIBRA and XENON100. The positive (negative) upper limit amplitude of $0.96(-1.5) \times 10^{-2}$ events $/ \mathrm{day} / \mathrm{kg} / \mathrm{keV}$ ee between 1.0 and $1.5 \mathrm{keV}_{\text {ee }}$ and $(1.3-3.2) \times 10^{-3}$ counts/day $/ \mathrm{kg} / \mathrm{keV}_{\text {ee }}$ between 2 and $6 \mathrm{keV}_{\text {ee }}$ were obtained. To find any period in the data, the power spectrum analysis was also performed with treating the phase $t_{0}$ free parameter, but no significant period was found between 20 and 600 days. As this analysis does not consider only nuclear recoils, a simple electron or gamma ray interpretation of the DAMA/LIBRA signal can also obey this limit.

\section{References}

[1] Y. Suzuki et al., [hep-ph/0008296].

[2] K. Abe et al. [XMASS Collaboration], Nucl. Instrum. Meth. A 716 (2013) 78

[3] Bernabei, R., Belli, P., Cappella, F. et al. Eur. Phys. J. C (2013) 73: 2648

[4] K. Abe et al. [XMASS Collaboration], Phys. Lett. B 759 (2016) 272

[5] [XMASS Collaboration], (to be published).

[6] E. Aprile et al., Phys. Rev. Lett. 107 (2011) 131302.

[7] M.C. Smith et al., Mon. Not. R. Astron. Soc. 379 (2007) 755-772.

[8] J.D. Lewin and P.F. Smith, Astroparticle Phys. 6 (1996) 87.

[9] J. Kopp et al. JCAP 03 (2012) 001.

[10] D.S. Akerib et al. (LUX collaboration), Phys. Rev. Lett. 118 (2017) 021303.

[11] E. Aprile et al. (XENON collaboration), [arXiv:1705.06655]

[12] R. Agnese et al. (SuperCDMS collaboration), Phys. Rev. Lett. 112 (2014) 241302.

[13] K. Abe et al. (XMASS collaboration), Phys. Lett. B 719 (2013) 78.

[14] C. E. Aalseth et al. (CoGeNT collaboration), Phys. Rev. D 88 (2013) 012002.

[15] R. Agnese et al. (CDMS collaboration), Phys. Rev. Lett. 111 (2013) 251301.

[16] E. Aprile et al. (XENON collaboration) Phys. Rev. Lett. 118 (2017) 101101. 